\title{
Acercamiento criminológico al estudio de la Delincuencia Económica en Granma - $\mathrm{Cuba}^{1}$
}

\section{Criminological approach to the study of economic crime in Granma - Cuba}

\author{
Omar Huertas Diaz \\ René Joaquin Martínez Gamboa ${ }^{3}$
}

Fecha de recepción: 12 de enero de 2016

Fecha de aprobación: 7 de marzo de 2016

\section{RESUMEN}

En la presente investigación se propone analizar la criminalidad económica y las distintas teorizaciones y conceptualizaciones que desde la criminología se han realizado alrededor de esta temática. Como se verá, los delitos económicos no encuentran fundamentos sociológicos o criminológicos en las razones dadas a la delincuencia tradicional. Estos delitos, también llamados de "cuello blanco", tienen determinadas características que los diferencian del resto de la delincuencia, y quienes los cometen no responden al patrón habitual del delincuente. Por ello,

1 Artículo en trabajo colaborativo resultado de Investigación del Grupo de Investigación "Escuela de Derecho Penal NULLUM CRIMEN SINE LEGE UN" de la Universidad Nacional de Colombia, Código COL0078909, Reconocido y Clasificado en categoría A COLCIENCIAS y Grupo de Investigación en Derecho Penal de la Universidad Gramma de Cuba. El artículo es producto del Proyecto de Investigación "Perspectiva criminológica del Sistema Penitenciario y Carcelario" con duración de 1 ańo y con código 23733 de la Universidad Nacional de Colombia y DEL GRUPO DE INVESTIGACIÓN EN DERECHO PENAL de la Universidad Gramma de Cuba.

2 Abogado, Profesor Asociado, Especialista en Derecho Penal, Líder Grupo de Investigación y Candidato a Doctor en Derecho, Universidad Nacional de Colombia, Ph.D en Ciencias de la Educación, Universidad Simón Bolívar. Mg. en Derecho Penal Universidad Libre, Máster en Derechos Humanos, Estado de Derecho y Democracia en Iberoamérica Universidad de Alcalá, España. Mg. en Educación Universidad Pedagógica Nacional. Socio de la Fundación Internacional de Ciencias Penales FICP. Miembro de honor de la Fundación de Victimología. Miembro Honorario Asociación Colombiana de Criminología. Correo electrónico: ohuertasd@unal.edu.co

3 Especialista de posgrado en Derecho Penal. Profesor Asistente de la disciplina de Ciencias Penales y Criminológicas del Departamento de Derecho en la Universidad de Granma - Cuba. E-mail: rmartinezg@udg.co.cu 
se toma como objetivo general caracterizar criminológicamente la delincuencia económica de la sociedad cubana actual, a partir de los estudios sociológicos realizados por varias instituciones al respecto. A tenor del problema científico que lo motiva, definido mediante la siguiente interrogante: ¿Cuáles son las principales características que presenta la delincuencia económica en la sociedad cubana actual? Utilizando a este fin los métodos siguientes: Método de Análisis y Síntesis, Análisis Lógico, Histórico Jurídico, Método Sociológico, Método Dialéctico Materialista. Como técnica de investigación fue empleada la revisión bibliográfica de los materiales que se consideraron más actualizados sobre el tema. Se presenta como fundamental resultado una caracterización del fenómeno de la delincuencia económica desde el punto de vista criminológico.

Palabras clave: criminología, Delincuencia Económica, sociedad cubana, delincuencia de cuello blanco, sistema económico.

\section{Abstract}

In this research, we propose to analyze the economic crime and the different theories and concepts that from criminology have been made about this subject. As we will see, economic crimes are not sociological or criminological basis in the reasons given to the traditional economic crime from the criminological point of view. These crimes, also called of "cuello blanco", have certain characteristics that differentiate them from the rest of the crime and the perpetrators do not respond to the usual pattern of the offender. So our principal objective is to characterize the economic crime in the current Cuban society, based on sociological studies conducted by various institutions in this regard. Under scientific problem that motivates, defined by the following question: What are the main characteristics that presents economic crime in Cuban society? Using for this purpose the following methods: Method of Analysis and Synthesis, Logical Analysis, Historical Legal, Sociological Method, Materialistic Dialectical Method. As research technique was used the literature review of materials we consider more updated on the subject. Presenting results in a fundamental characterization of the phenomenon of economic crime from the criminological point of view.

Keywords: Criminology, economic delinquency, cuban society, delinquency of "cuello blanco", economic sistem. 


\section{INTRODUCTION}

La delincuencia económica es tan antigua como la humanidad misma. La historia así lo demuestra desde que unos se enriquecieron a costa de otros, muchas veces de forma fraudulenta y utilizando el poder. Este fenómeno comenzó a manifestarse aun sin la explicación científica oportuna.

La historia del estudio científico de la delincuencia económica, sin embargo, es más reciente. Sin lugar a dudas, se puede afirmar que un particular aspecto de la misma es el análisis de este tipo de delincuentes, como lo hace Edwin Sutherland en su famosa obra "El delincuente de Cuello Blanco". No obstante, los antecedentes en el pensamiento criminológico pueden mostrarse mucho antes a partir de autores que podrían asombrar por su percepción temprana acerca de este asunto. Es así como Solón (Plutarch, trad. 1960), legislador de Atenas, manifestó en sus poesías apreciaciones éticas acerca de algunos comerciantes: "A menudo los malvados prosperan, mientras los justos mueren de hambre” (p. 45).

En el estudio de la Criminología, resalta Lombroso (Trad. 1899), fundador de esa disciplina como ciencia y a quien se le ha vulgarizado, no tanto por lo que dijo o lo que se dice que dijo sino por las limitaciones o la ignorancia en el conocimiento de su obra; un precursor, un anunciador de este fenómeno. Ese autor decía: "El hombre de estado que desea prevenir el delito debe protegerse de los efectos peligrosos de la riqueza en la misma medida que de los de la pobreza" (p. 181).

En la actualidad, la distinción que interesa es otra. No se trata de lo patrimonial sino de lo "económico". Pero la cuestión reconoce rangos de consideración semejantes: va desde la aplicación de los más severos castigos a la aceptación como travesura simpática de ciertos hechos calificados de delitos económicos.

"Se deslinda el sentido estricto y el amplio tanto del Derecho Penal Económico como del delito económico. El primero es el que atiende a la intervención del estado en la economía, el segundo a la regulación jurídica de la producción, distribución y consumo de bienes y servicios. La contraposición, según él, es entre el intervencionismo estatal por un lado y la economía de mercado por el otro" (Bajo Fernández, 1978. p. 37). 
En la presente investigación se propone analizar la criminalidad económica y las distintas teorizaciones y conceptualizaciones que desde la criminología se han realizado alrededor de esta temática. Como se verá, los delitos económicos no encuentran fundamentos sociológicos o criminológicos en las razones dadas a la delincuencia tradicional. Estos delitos, también llamados de "cuello blanco", tienen determinadas características que los diferencian del resto de la delincuencia, y quienes los cometen no responden al patrón habitual del delincuente. Asimismo, las sanciones para este tipo de delitos tienen matices distintivos. Por otra parte, aunque provocan enormes daños económicos y cuantiosas pérdidas, que pesan sobre el conjunto de la sociedad, el delito de "cuello blanco" es percibido como oculto o invisible. En primer lugar, se emprenderá la tarea de conceptualizar qué se entiende por delitos económicos, que hoy se percibe como uno de los aspectos más deficientes de la operatividad penal. El delito de "cuello blanco" es definido por Sutherland (como se citó en Aller, 2005) como "un delito cometido por una persona de respetabilidad y de 'status' social alto en el curso de su ocupación". Las claves de estos conceptos son el prestigio social y el poder: estas personas no son vistas como delincuentes.

Quienes cometen estos delitos no responden al concepto de delincuente tradicional, definido desde lo patológico y proveniente de sectores desprotegidos socialmente. Por el contrario, los delincuentes de "cuello blanco" están plenamente "integrados" a la sociedad, situación que lleva a pensar que estos delitos, lejos de constituir una conducta desviada, son parte del funcionamiento de un determinado sistema económico -el capitalismo-, que se apoya en esta delincuencia y esta al mismo tiempo se fundamenta y encuentra lazos profundos en determinados sectores de la economía. La criminalidad económica puso de manifiesto el equívoco de las teorías criminológicas tradicionales que siempre explicaron el crimen a partir de la dicotomía orden y desorden. El delincuente de "cuello blanco" es un respetable hombre de negocios, quien no se percibe a sí mismo como tal, usa técnicas de neutralización que permiten enmascarar la conducta ilícita bajo el aspecto de prácticas comerciales comunes y corrientes. Uno de los rasgos distintivos de la criminalidad económica es la impunidad e inmunidad con que se manejan los autores, quienes pertenecen a estratos sociales privilegiados, tienen contactos con personas poderosas y utilizan medios no violentos. Por otra parte, existe gran dificultad en la percepción y prueba del delito. 
Para iniciar el estudio sobre el tema debemos plantear el problema científico que lo motiva, definido mediante la siguiente interrogante: ¿Cuáles son las principales características que presenta la delincuencia económica en la sociedad granmense actual? El cual genera la siguiente hipótesis: no existe una clara caracterización de la delincuencia económica en ese territorio que permita un estudio eficiente del fenómeno desde la óptica de la criminología en busca de un eficaz enfrentamiento. Teniendo en cuenta lo antes expuesto, resulta procedente como objetivo general caracterizar a partir de un estudio teórico, histórico y criminológico la delincuencia económica de la sociedad granmense en pos de su prevención y enfrentamiento en correspondencia con su dinámica actual. Se derivan así tres objetivos específicos:

- Delimitar la relación existente entre la delincuencia económica e instituciones tradicionalmente relacionadas a esta dentro de la dinámica de esta categoría.

- Determinar los principales referentes históricos de la delincuencia económica en Cuba, de manera que permita un acercamiento a las características de este fenómeno en el contexto actual en la provincia Granma.

- Identificar los rasgos distintivos de la delincuencia económica en Granma, para contribuir a la prevención y enfrentamiento de este fenómeno en el territorio.

Los métodos utilizados en la investigación para dar respuesta al problema planteado fueron:

- Método de Análisis y Síntesis: para sintetizar todo el contenido estudiado para la investigación.

- Análisis Lógico: para ofrecerle un orden lógico a la estructura del trabajo a partir de la información consultada.

- Histórico Jurídico: para buscar los orígenes y observar la evolución de cada una de las instituciones jurídicas abordadas. 
- Método Sociológico: para valorar el papel que ha de atribuirse a la delincuencia económica en las sociedades modernas y de la posición, que como tendencia ha de ocupar esta en el estudio criminológico del fenómeno delincuencial.

- Método Dialéctico Materialista: para comprender las verdaderas causas que han generado la evolución constante de la delincuencia económica en nuestra sociedad socialista

- Método Empírico: para constatar las inferencias teóricas expuestas en la investigación.

- Como técnica de investigación fue empleada la revisión bibliográfica de los materiales que se consideraron más actualizados sobre el tema objeto de la investigación, así como la encuesta a sujetos comisores de estos delitos, la entrevista a expertos con reconocida experiencia en el enfrentamiento e investigación sobre el tema analizado y el estudio de casos representativos dentro de este tipo de delincuencia.

La investigación se estructuró a partir de la metodología de la triangulación, incorporando los siguientes tipos:

Triangulación teórica: que permitió la utilización de perspectivas múltiples para analizar lo concerniente a la delincuencia económica.

Triangulación metodológica: cohesionó diferentes métodos para la solución del problema trazado.

La metodología triangulada posibilitó no solo dotar a la investigación de validez interna, pues en la recogida de información e interpretación fueron utilizados diferentes métodos y perspectivas, sino también de validez externa, pues la propuesta de caracterización criminológica de la delincuencia económica en Granma, que constituye la principal recomendación, fue sometida y corroborada por otros juristas vinculados al ejercicio profesional, como se explicará más adelante. 


\section{Desarrollo}

\section{Delincuencia económica en la sociedad contemporánea actual}

A pesar de que existen vínculos innegables entre la delincuencia económica y el sistema capitalista, no es posible, científicamente, considerar que la criminalidad económica es un comportamiento exclusivo de las sociedades occidentales. También en los sistemas socialistas existe una criminalidad económica específica, cuya impunidad se garantiza mediante el abuso de poder y la corrupción (Günther, 1978; López-Rey Arrojo, 1975). Así como en occidente la delincuencia económica se caracteriza por los acuerdos monopolísticos, la competencia ilícita, el espionaje industrial y los delitos societarios, en los países socialistas, esta criminalidad se expresa en la corrupción administrativa, las falsedades económicas, el mercado negro y la lesión de los planes económicos o las normas de producción (Zemtsov, 1977).

El concepto de delincuencia económica se traduce en un eufemismo, adoptado doctrinalmente para definir a los ilícitos de orden penal que varios ordenamientos penales denominan con el rubro de delitos patrimoniales. Los delitos de "cuello blanco" dentro de los cuales encuadran los delitos económicos no dañan únicamente a la persona física o moral que sufre un menoscabo en su patrimonio, sino también van en contra de la economía del Estado y por ende del orden público económico interno y global.

Otro de los factores trascendentes de la delincuencia económica para el análisis económico del derecho es que no se trata del delincuente común: se habla de sujetos con capacidades y adiestramiento profesional, lo que trae aparejado la desestimación de la trascendencia social, jurídica y política de las consecuencias de la realización de este tipo de delitos.

Este tipo de delitos tuvo primeramente un interés analítico, sociológico y criminológico, para luego ser objeto de estudio ante las posibilidades que ofrecía en la lucha contra la criminalidad económica. Sin embargo, esta temática no se extendió igualmente a todas las regiones y países. En Europa, cobra vida fundamentalmente en la entonces República Federal de Alemania, en los años 70, cuando se comienza 
a desarrollar un análisis crítico de los medios e instrumentos legales con los que contaba dicho país para hacer frente a la creciente criminalidad económica que había sido fertilizada con la reconstrucción emprendida en las décadas de los años 50 y 60 de toda la infraestructura destruida en la Segunda Guerra Mundial, pero que no había otorgado igual preponderancia a la moral económica.

Se hace necesario, entonces, conceptualizar los delitos económicos para poder comprender su alcance y contenido. Al respecto, Puig Peńa (como se citó en Grillo Longoria, 1982) exponía que eran "los que quebrantan la vida económica de la sociedad en general, ya que trastocan, por así decirlo, la economía del país”. Un criterio criticado con acierto por Grillo Longoria (1982) al decir "que hacía creer que en la sociedad capitalista los intereses económicos de la sociedad son armónicos, que son los mismos para el explotador que para el explotado" (p. 310).

En una concepción más progresista, Pérez (1967) considera:

"Delito Económico es la violación del derecho que asiste a los asociados, sin distinción de clases, para beneficiarse con los elementos de la naturaleza y los bienes creados por el trabajo, ocasionada con ánimo de destrucción o de lucro" (p. 158).

Grillo Longoria (1982) refiere que son "aquellos hechos atentatorios contra la formación económico-social de un país, que producen un grave daño o representan un peligro grave" (p. 312).

Entretanto, para Baigún (1995):

"El delito económico es el comportamiento con resultado dañoso social que lesiona directamente el orden económico, entendido este como un bien supraindividual o colectivo; cabe tanto el que se halla incorporado a la legislación, como el desaprobado socialmente que reclama su tipificación” (p. 126). 
En este concepto, señala el autor, que los sujetos de la acción pueden ser el particular o la persona jurídica, aclarando que en el momento actual el ente colectivo ha pasado a tener un rol decisivo.

Aun cuando en estos conceptos, se ponen de manifiesto diferencias que tienen de base la sociedad que se representa. En todos está presente el perjuicio que tales actos ocasionan a la economía de un país, por encima de los derechos concretos de los individuos o como bien supraindividual, en los que se admiten no solo a los que provienen de la propia actividad económica, relacionados con la producción, distribución y consumo de bienes, entre ellos los que lesionan el mercado cambiario, la inversión de capitales y la hacienda pública, sino además los delitos denominados societarios.

Realizar una periodización de este fenómeno puede situar en cuáles han sido sus principales momentos de cambio y la forma en que se ha desarrollado la creación normativa para darle respuesta a esa evolución continua.

\section{Análisis general y validación de los resultados}

Uno de los métodos que se utilizó para validar las propuestas de solución al problema investigado fue el método de consulta de expertos con procesamiento estadístico de los datos.

Para ello, se aplicó una entrevista a un grupo de expertos previamente seleccionados de especialistas avezados sobre el fenómeno o tema que se analiza, quienes brindaron información necesaria sobre la validación de las propuestas elaboradas, recurriéndose a un tratamiento matemático-estadístico, a fin de delimitar su certeza y fiabilidad. A los efectos de su aplicación, se considera experto a aquel especialista cuyos conocimientos, experiencias, desarrollo intelectual y capacidades para prever tendencias o condiciones futuras le permiten brindar una información útil sobre cierto problema o fenómeno. La confiabilidad de los resultados que se obtengan con la aplicación de este método dependerá en gran medida de tres factores esenciales:

- El número de expertos. 
- El rigor en la selección de los expertos.

- La exactitud de los procedimientos estadísticos utilizados.

Primera etapa: determinación del número de expertos. Existen varios criterios alrededor de la cantidad óptima de expertos a consultar. Se decidió acoger el criterio expuesto por Silva y Suanes (1986), autores que, para obtener el número óptimo de expertos a consultar, buscan una correlación entre la cifra de expertos y el error medio grupal. Otros autores consideran diferentes cifras en lo concerniente al número adecuado de expertos a consultar, como Celis Mestre (2002), quien señala que la cifra adecuada es entre 10 y 13 expertos.

De acuerdo con la curva que se obtiene (ver Anexo 2), se puede concluir que las cifras más apropiadas de expertos oscilan entre 17 y 25. Por debajo de 17 expertos, se incrementa el riesgo de error y, por encima de 25 , no se obtiene incremento significativo en la fiabilidad de las opiniones. En el caso de la presente investigación se consultaron un total de 21 expertos para realizar la consulta y validación de los resultados.

Segunda etapa: el rigor en la selección de los expertos. Un primer elemento que se consideró fue la voluntariedad de los participantes, para luego pasar a la determinación de las competencias de estos, la cual expresa su nivel de calificación en el tema y problema de la investigación. Sobre la determinación de la competencia de los expertos, se plantean diferentes vías. Se adoptó el criterio de selección de competencia a partir de la opinión generalizadora acerca de la autoridad del especialista en el problema investigado (Celis Mestre, 2002). Una de las variantes más utilizadas en estos casos es la determinación del coeficiente de competencia del experto $(\mathrm{k})$; el cual se calcula a partir de la autoevaluación realizada por el experto acerca del nivel de conocimiento sobre el tema o problema en la siguiente fórmula: $\mathrm{k}=1 / 2(\mathrm{kc}+\mathrm{ka})$, donde $(\mathrm{kc})$ es el coeficiente de conocimiento o información que tiene el experto y $(\mathrm{ka})$ es el coeficiente de argumentación o fundamentación de los criterios del experto sobre el tema.

Los indicadores que se tuvieron en cuenta en esta ocasión fueron los siguientes: 
- La experiencia en el enfrentamiento al problema investigado (más de cinco años).

- El dominio teórico sobre el problema investigado (en este indicador se valoró el nivel científico e investigativo del experto y si realizó con anterioridad investigaciones relacionadas con la problemática investigada).

- Jerarquía institucional. Está referido al rol que ocupa el experto dentro de una estructura organizacional relacionada con el ejercicio del derecho, el cual le permite poseer una visión global del problema investigado.

Los expertos seleccionados ascienden a 21, los cuales poseen las siguientes características (ver Anexo 1). El promedio de años de experiencia entre los expertos seleccionados es de 17,7 años.

Tercera etapa: elaboración, aplicación y procesamiento estadístico de los resultados obtenidos. Después de elaborada la encuesta aplicada (ver Anexo 4), en la cual se recogen todos los indicadores expuestos tanto en el modelo teórico como en las propuestas de solución, se procedió a entregárselo a los expertos seleccionados. Los datos obtenidos son cualitativos y los procedimientos estadísticos constituyen el instrumento que posibilitó su análisis, para lo cual a cada dato ofrecido se le asignó un valor numérico. Dentro de estos procedimientos se utilizó la media para determinar el promedio de los diversos valores clasificados por categorías, la desviación estándar para establecer la oscilación que mostró las diversas opiniones vertidas por los expertos respecto a la media, la moda establece cuál es el criterio de los expertos que más se repite por serie, el coeficiente de concordancia de las opiniones de los expertos posibilitó establecer el grado de coincidencia de estas respecto a un rango de puntuaciones y el cálculo porcentual brindó la significación porcentual de las diferentes opiniones recogidas, es decir, su representación específica (el \%) dentro del total de expertos consultados (100\%). Para ver las fórmulas empleadas ver el Anexo 3.

Partiendo de los métodos por los cuales se arribó a la elaboración de la caracterización de la delincuencia económica en el territorio y las propuestas de enfrentamiento, 
los efectos de la aplicación del método de consulta de expertos con procesamiento estadísticos de los datos y que los resultados a que aporta la doctrina, como se demostró supra (Capítulo 1), coinciden en parte con los aquí propuestos. Se puede arribar a la conclusión de que son válidos los resultados expuestos y pueden implementarse en la práctica jurídica del territorio en aras de contribuir a su eficaz enfrentamiento. Son estos los siguientes:

Los delitos económicos se han incrementado en la provincia en número de hechos y coautores o partícipes de modo proporcional con su persecución, partiendo de los grupos de análisis y los delitos infraganti.

Las principales causas son la negligencia de los administrativos que propician que otros funcionarios se apropien de los bienes, por no cumplir con las conciliaciones y verificaciones económicas. Insuficientes controles económicos, además de la no existencia de contrapartida en el ejercicio de las funciones y vulneración de las normas de contabilidad. En el sector cooperativo existe deficiente funcionamiento del aparato económico. Por otro lado, incumplen la Resolución número 2253/ 2005 del Ministerio de Economía y Planificación, que establece las indicaciones para la Contratación Económica, sobre la forma, lugar y tiempo de entrega de las mercancías, lo que se utiliza para cometer los hechos delictivos. Se incumple con el Control Interno establecido por la Resolución número $60 / 11$ de la Contraloría General de la República en el componente Actividad de Control en la norma Coordinación entre Áreas, separación de tareas, responsabilidades y niveles de autorización.

Modus operandi: los más usados son la falsificaciones para defraudar, doble facturación, transferencias ficticias de los recursos, método de centrifuga (es la forma en la que van acumulando efectivo de las ventas realizadas, no lo depositan todo, se quedan con una parte y realizan la documentación). Al ponerse de acuerdo directivos de las entidades involucradas, se efectúan los pagos correspondientes para evitar que se detecten los hechos. En casos de actividades relacionadas con la producción de café, arroz y porcino, lo común es que se declaren producciones ficticias para efectuar el pago a los supuestos productores y se reparten las ganancias obtenidas. No se realizan los depósitos del efectivo correspondientes a las ventas realizadas y se centrifuga el mismo, lo que permite apropiarse del dinero. 
El enfrentamiento se ha fortalecido indiscutiblemente en el territorio, tal como lo demandan las prioridades del país y lo ha hecho sobre todo con el fortalecimiento del control interno y el actuar de la Contraloría General de la República, pero es deficiente y se retarda el enfrentamiento oportuno, por lo que aún no rinde los frutos esperados.

Los acusados generalmente aprovechan sus cargos y funciones relacionados con la custodia, disponibilidad y administración de los bienes y se apoyan en otras personas para llevar a vías de hechos sus objetivos los que se convierten en cómplices o receptadores. Aprovechan generalmente la falta de control y fiscalización. Los acusados de este tipo de delitos en un $90 \%$ son primarios en la comisión de hechos delictivos y el $10 \%$ restante corresponde a reincidentes específicos, pero con sanciones subsidiarias anteriores que impiden su apreciación. Por lo general, son universitarios o de la categoría de técnicos. Personas que oscilan entre los 25 y 50 años de edad, predominan los hombres, aunque se aprecia un ligero incremento de la participación de mujeres sobre todo como cómplices o encubridoras.

Los delitos que más inciden son Malversación y Apropiación Indebida. Como tendencia, se ha podido evidenciar el incremento de la ocultación u omisión de datos por la desaparición de la documentación en el momento en que se ejecuta la acción de control y el crecimiento en los niveles de organización delictiva, así como en la cuantía de las afectaciones al orden económico. 


\section{Conclusiones}

Primera: los criterios para analizar la delincuencia están dados por la interrelación de tres categorías fundamentales. En primer lugar, por el estado en que esta se encuentra, dígase valores cuantitativos de la delincuencia, época, zona y proporción. En segundo lugar, por su estructura contentiva de características, tipos de delitos, edades, sexos y, por último, de su dinámica, que se expresa en las relaciones que se establecen entre estado y la estructura que exhibe la delincuencia.

Segunda: la delincuencia económica, la delincuencia de "cuello blanco" y la delincuencia organizada se distinguen esencialmente en que la primera hace referencia únicamente a la generalidad de los delitos económicos no exigiendo requisitos adicionales en su configuración; mientras que la segunda agrega a esto una posición social privilegiada por parte del sujeto comisor y de igual modo la tercera incorpora criterios de orden a un nivel superior con fines y métodos distintos.

Tercera: se aprecia que en Cuba después de 1959 la delincuencia económica tendencialmente ha ido perfeccionando sus métodos y el nivel de organización ha crecido, representando esto mayores afectaciones a la economía y, a su vez, cierto descrédito en cuanto a la gestión de algunos actores públicos inmersos en este fenómeno delincuencial. Aparejado esto a las transformaciones económicas que vive nuestro país, es posible prever un aumento de este tipo de delincuencia.

Cuarta: entre las principales características que presenta la delincuencia económica en la sociedad granmense actual, se destacan las relativas a:

\section{Los autores:}

- quienes como generalidad se distinguen como materialistas, inteligentes, egocéntricos.

- generalmente aprovechan sus cargos y funciones relacionados con la custodia, disponibilidad y administración de los bienes. 
- por lo general son universitarios o de la categoría de técnicos.

- oscilan entre los 25 y 50 años de edad.

- predominan los hombres, aunque se aprecia un ligero incremento de la participación de mujeres sobre todo como cómplices y/o encubridoras.

\section{Modus operandi:}

- sobrefacturación y la subfacturación de productos.

- las falsificaciones para defraudar.

- doble facturación.

- transferencias ficticias de los recursos.

- se declaran producciones ficticias, para efectuar el pago a los supuestos productores y se reparten las ganancias.

- método de centrífuga.

- no se realizan los depósitos del efectivo correspondientes a las ventas realizadas y se centrifuga el mismo.

\section{Principales causas:}

- negligencia de los administrativos que propician que otros funcionarios se apropien de los bienes por no cumplir con las conciliaciones y verificaciones económicas.

- insuficientes controles económicos, además de la no existencia de contrapartida en el ejercicio de las funciones y vulneración de las normas de contabilidad. 
- en el sector cooperativo existe deficiente funcionamiento del aparato económico.

\section{Sectores más afectados:}

- están relacionados con la producción de café, arroz y porcino.

Quinta: El enfrentamiento se ha fortalecido indiscutiblemente en el territorio, tal como lo demandan las prioridades del país, y lo ha hecho sobre todo con el fortalecimiento del control interno y el actuar de la Contraloría General de la República, pero es deficiente y se retarda el enfrentamiento oportuno, por lo que aún no rinde los frutos esperados, lo que demuestra que deben perfeccionarse los métodos y aparatos de control para con el conocimiento de las reales dimensiones del fenómeno, enfrentarlo eficazmente.

\section{RECOMENDACIONES}

1. A la Fiscalía Provincial de Granma, al Tribunal Provincial Popular de Granma y a la Delegación del MININT en Granma:

- Utilizar la caracterización aportada en la investigación para delimitar y proyectar la política penal con respecto a estos delitos para lograr un enfrentamiento eficaz a los actos delictivos contra el orden económico.

2. Al Comité Provincial de los CDR y a la FMC Provincial de Granma:

- Utilizar los resultados de esta investigación fundamentalmente en la comprensión del fenómeno y la dimensión que este alcanza hoy en nuestra sociedad con fines de trabajo preventivo.

3. A los órganos de enfrentamiento al delito en la provincia Granma: 
- Que a partir de la información obtenida sobre las características de este tipo de delincuencia se creen nuevas formas y metodologías de enfrentar el delito económico en la provincia por la importancia que esto revierte, sobre todo en momentos de transformaciones económicas significativas como el que se encuentra inmerso nuestro país.

4. A la Facultad de Derecho de la Universidad de Oriente, a la carrera de Derecho de la Universidad de Granma y a la Unión de Juristas de Cuba:

- Contribuir a través de cursos de superación a la formación de los operadores jurídicos respecto a las características de este tipo de delincuencia en Cuba.

- Continuar realizando investigaciones asociadas al tema teniendo en cuenta la importancia que reviste en el contexto actual. 


\section{REFERENCIAS}

Aller, G. (2005). White collar crime. Edwin Sutherland y el Delito de Cuello Blanco. Revista de Derecho Penal y Procesal Penal, (2012). p. 31 - 53. Edición julio - Diciembre.

Baigún, D. (1995) Tendencias actuales del derecho penal económico en América Latina. Necesidad de un nuevo modelo. Revista Cubana de Derecho, (11). p. 126.

Bajo, M. (1978). Derecho Penal Económico aplicado a la actividad empresarial. Madrid: Civitas.

Celis, F. (2002). Método Delfos. En Félix Varela (Ed.), Colectivo de Autores: Ordenando el caos. La Habana: Editorial Maestre. pp. 50-78.

Grillo, J. A. (1982). Los Delitos en Especie. Cuba: Editorial Maestre.

Günther, K. (1978). Criminología. España: Espasa Calpe.

Lombroso, C. (1899). Crimen, sus causas y remedios. Cuba: Editorial Maestre.

López, M. (1975). Criminología. España: Ed. Jurídica Aguilar.

Pérez, L.C. (1967). Tratado de Derecho Penal. T. 4. Bogotá: Temis.

Plutarch (1960). The Rise and Fall of Athens. Nine Greek Lives. Trad. de Scott Clivert, I. Baltimore: PenguinBooks.

Silva, M. y Suanes, H. (1986). El método de las evaluaciones de los expertos y su aplicación en la confección de planes de estudio. La Habana: Academia Superior de las FAR "General Máximo Gómez".

Zemtsov, I. (1977). La corrupción en la Unión Soviética. España: Ed. Plaza \& Janés, SA. 


\section{ANEXOS}

\section{Anexo 1. Listado de los Expertos consultados}

1. Dr. Rodolfo Máximo Fernández Romo. Profesor Titular de Criminalística de la Facultad de Derecho de la Universidad de la Habana, 25 años de experiencia.

2. Capitán Yadimé Aurora Hernández López. Segunda Jefa de Unidad UPICO Granma, Instructora penal, miembro del grupo económico, 19 años de experiencia.

3. Primer Teniente Urisbel Hernández Pérez. Instructor penal UPICO Granma, miembro del grupo económico, 8 años de experiencia.

4. Capitán Ernesto Figueredo Castillo. Instructor penal UPICO Granma, miembro del grupo económico, 25 años de experiencia.

5. Capitán Amaury Ricardo Rivero. Instructor penal UPICO Granma, Primer Instructor del grupo económico, 25 años de experiencia.

6. Mayor Ladihe Ernesto Boza Cambar, Oficial operativo CIM, 17 años de experiencia.

7. Especialista el Derecho Haydee Aponte Tornés. Juez Titular de la Sala Segunda de lo Penal del Tribunal Provincial Popular de Granma, 25 años de experiencia.

8. Especialista el Derecho Penal Alejandro Vázquez Almenares. Juez Titular de la Sala Primera de lo Penal del Tribunal Provincial Popular de Granma, 6 ańos de experiencia.

9. Reinaldo Roldan Peña. Juez, Presidente del Tribunal Municipal de Guisa, 14 años de experiencia. 
10. Primer Teniente Juan Alberto Simón Vega. Primer Juez del Tribunal Militar Región Granma, 7años de experiencia.

11. Especialista el Derecho Penal José Alexis Ginarte Gato. Abogado del Bufete colectivo Bayamo, Presidente de la UNJC en Granma, 20 ańos de experiencia.

12. Especialista el Derecho Penal Alexis Aurelio Hechavarría. Abogado del Bufete colectivo Bayamo, 25 años de experiencia.

13. Especialista el Derecho Penal Yenisey González Rodríguez. Fiscal del Departamento Provincial de Procesos Penales, 14 años de experiencia.

14. Especialista el Derecho Penal Osvaldo Rivero Meireles. Fiscal del Departamento Provincial de Procesos Penales, 33 años de experiencia.

15. Especialista el Derecho Penal Rudy Castillo Rosa. Fiscal del Departamento Provincial de Procesos Penales, 17 años de experiencia.

16. Especialista el Derecho Penal Elides Barbán Gómez. Fiscal del Departamento Provincial de Procesos Penales, 15 años de experiencia.

17. Especialista el Derecho Penal Magalis Figueredo Molina. Fiscal del Departamento Provincial de Procesos Penales, 30 ańos de experiencia.

18. Especialista el Derecho Penal Cecilia Teresa Reyes Trujillo. Fiscal del Departamento Provincial de Procesos Penales, 25 años de experiencia.

19. Julio Cabrera Leyva. Fiscal del Departamento Provincial De Procesos Penales, 17 años de experiencia.

20. Lisandra León Brizuela. Psicóloga, Jefa de la Carrera de Psicología en la Universidad de Granma y Psicóloga de medicina Legal, 11 años de experiencia. 
21. Mirelle Santoya Rosado. Socióloga, Contraloría General de la República en Granma, 10 años de experiencia.

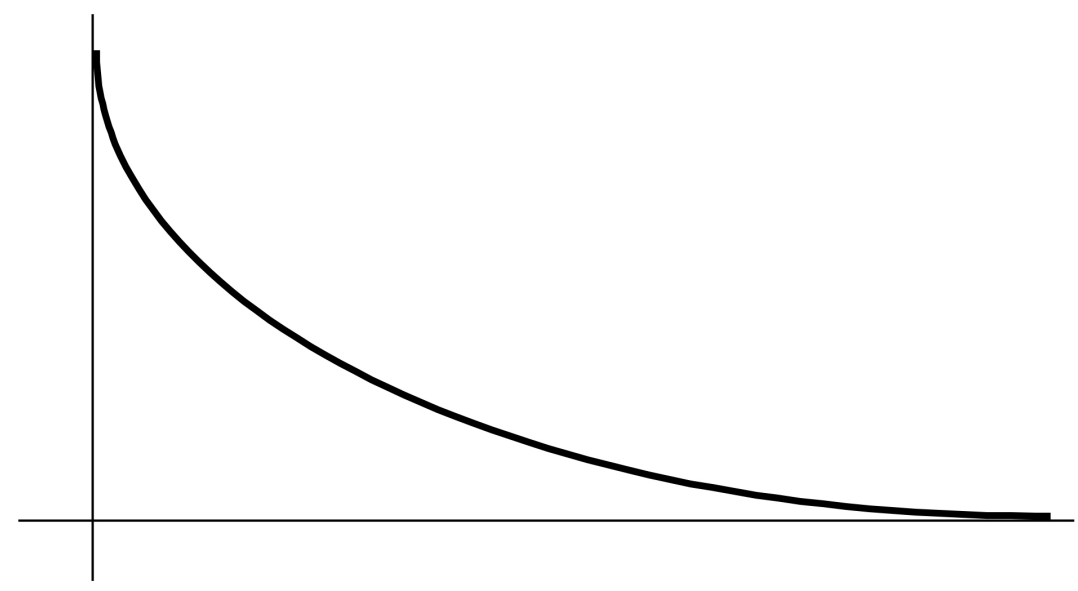




\section{Anexo 2. Error medio grupal}

Error medio grupal.

1.2

1.1

1.0

0.9

0.8

0.7

0.6

0.5

0.4

12345678910111213141516171819202122232425

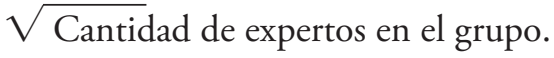

Interdependencia entre la cantidad de expertos en el grupo y el error medio grupal. 


\section{Anexo 3. Fórmulas}

Desviación estándar $(S): S=S^{2}$, donde $S^{2}$ es la varianza promedio que se obtiene de sumar las desviaciones al cuadrado de cada dato con relación a la media de la serie y dividida ésta, por la suma del total de observaciones.

Coeficiente de concordancia (V): $V=S \times 100$

\section{$\mathrm{X}$}

$\mathrm{X}$ es la sumatoria del valor de los datos sobre la cantidad de datos.

Moda (Mo): $M o=R 1+\mathrm{N}_{\mathrm{p}} x \mathrm{I}$

$$
\mathrm{N}_{\mathrm{a}}+\mathrm{N}_{\mathrm{p}}
$$

$\mathrm{R} 1$ es el límite real inferior, $\mathrm{N}_{\mathrm{p}}$ es la frecuencia posterior a la frecuencia modal, $\mathrm{N}_{\mathrm{a}}$ es la frecuencia anterior a la frecuencia modal e I (intervalos) $=\mathrm{R}_{\mathrm{s}}$ (Límite superior) $-\mathrm{R}_{1}$. 


\section{Anexo 4}

\section{Encuesta A Expertos}

Experto No.

Estimado (a) especialista:

La presente encuesta tiene como objetivo, recoger sus opiniones sobrela caracterización de la delincuencia económica en el territorio y las propuestas de enfrentamiento, las cuales son producto de una investigación sobre el estudio criminológico de la delincuencia económica en Granma. Su opinión nos resultará de gran valía, ya que permitirá perfeccionar y validar las propuestas sometidas a su consideración.

Muchas Gracias

\section{Datos del Experto}

Profesión

Grado Científico $\longrightarrow$ Categoría Docente

Organismo a que pertenece

Años de experiencia profesional

Ha impartido clases de: pregrado $(\mathrm{x})$ postgrado (x)

\section{Contenido de la Validación}

La tabla que le presentamos a continuación está referida al modelo teórico elaborado por el autor de la investigación para argumentar y sustentar, las propuestas de solución a las que arribó. 
I. Marque con una (x) la alternativa que considere pertinente en cada uno de los siguientes elementos:

\begin{tabular}{|l|c|c|c|c|c|} 
Indicadores a valorar & $\begin{array}{c}\text { C1 } \\
\text { muy } \\
\text { adecuado }\end{array}$ & $\begin{array}{c}\text { C2 } \\
\text { bastante } \\
\text { adecuado }\end{array}$ & $\begin{array}{c}\text { C3 } \\
\text { adecuado }\end{array}$ & $\begin{array}{c}\text { C4 } \\
\text { poco } \\
\text { adecuado }\end{array}$ & $\begin{array}{c}\text { C5 } \\
\text { no } \\
\text { adecuado }\end{array}$ \\
\hline
\end{tabular}

Características

de los autores

Principales causas

\section{Modus Operandi}

\section{Sectores más afectados}

\section{Caracterización}

general

II. Marque con una (x) en la escala del 0 al 10, siendo el 10 la máxima puntuación que le otorga a la caracterización general descrita en la investigación.

\begin{tabular}{|l|l|l|l|l|l|l|l|l|l|l|}
\hline 0 & 1 & 2 & 3 & 4 & 5 & 6 & 7 & 8 & 9 & 10 \\
\hline
\end{tabular}

